\title{
Crystal structure analysis of L-asparaginase from Thermococcus litoralis DSM 5473
}

Tomomi Fujii ${ }^{1}$, Takae Yamauchi ${ }^{1}$, Shiro Kato ${ }^{2}$, Tadao Oikawa ${ }^{3}$, Yasuo Hata ${ }^{1}$

${ }^{1}$ Institute For Chemical Research, Kyoto University, Uji, Japan, ${ }^{2}$ International Institute of Rare Sugar Research and Education, Kagawa University, Kita-gun, Japan, ${ }^{3}$ Faculty of Chemistry, Materials and Bioengineering, Kansai University, Suita, Japan E-mail: fujii@scl.kyoto-u.ac.jp

L-Asparaginase (E.C. 3.5.1.1) is an enzyme that catalyzes the hydrolysis of L-asparagine to L-aspartate and ammonia. The enzyme has been used as an effective antineoplastic agent for acute lymphoblastic leukemia. In food industry, it is expected as a food processing aid to reduce acrylamide levels in cooked food. Thermococcus litoralis DSM 5473, which was isolated from a hot spring of a shallow sea, is a hyperthermophilic archaeon and has the ability to grow optimally at $88{ }^{\circ} \mathrm{C}$. In order to study the industrial utilization of thermostable L-asparaginase, the gene encoding the enzyme from T. litoralis DSM 5473 (TIASNase) was cloned and expressed in Escherichia coli. TIASNase has a distinctive substrate specificity; the enzymatic activity to D-Asn is $70 \%$ of that to L-Asn whereas other reported L-asparaginases have no or low activity to D-Asn. To elucidate the structural basis for the catalytic mechanism, substrate recognition, and thermostability, we analyzed the crystal structure of TIASNase. The enzyme was crystallized at $20^{\circ} \mathrm{C}$ by the sitting-drop vapour-diffusion method using $25 \%$ ( $/ \mathrm{v}$ ) 2-propanol, $0.25 \mathrm{M}$ ammonium acetate, and $0.1 \mathrm{M}$ Tris- $\mathrm{HCl} \mathrm{pH} \mathrm{8.5.} \mathrm{Diffraction} \mathrm{measurement} \mathrm{was} \mathrm{performed} \mathrm{at} \mathrm{beamline} \mathrm{BL-}$ 17A, Photon Factory, Tsukuba, Japan. During the measurement the crystal soaked in crystallization solution containing $30 \%$ $(\mathrm{v} / \mathrm{v})$ glycerol was cooled in a nitrogen stream $100 \mathrm{~K}$. The crystal belonged to space group P6122 with unit cell parameters a $=\mathrm{b}=69.55, \mathrm{c}=558.15 \AA$ and diffracted to $2.3 \AA$ resolution. The structure was determined by molecular replacement and refined at $2.3 \AA$ resolution. TIASNase is a homodimer, existing in the asymmetric unit of the crystal. The subunit has 331 amino acid residues and consists of two domains connected by a linker region of 20 amino acid residues. The $\mathrm{N}$-terminal domain of 185 amino acid residues adopts a large $a / \beta$-structure, which comprises four $a$-helices and twelve $\beta$-strands. Its core structure is flavodoxin-like fold with four $\alpha$-helices and eight $\beta$-strands. The C-terminal domain of 126 amino acid residues has a small $a / \beta$-structure, which has a parallel $\beta$-sheet of four $\beta$-strands with five a-helices. The TIASNase dimer is formed mainly by intersubunit interactions between $\beta$-strands of $C$-terminal domains, resulting in the formation of the large intersubunit eight stranded $\beta$-sheet. From the amino-acid sequence alignment and structural comparison with related enzymes, Thr12 and Thr86 in TIASNase are supposed to be catalytic residues. The structural comparison of active sites shows that the two portions of the loop regions in the active site have different conformations from those of related enzymes. These sites are candidates for the recognition sites to substrates. The trials of the crystallization of the enzymesubstrate/product complexes are under way.

Keywords: asparaginase, thermostability, D-amino acid 